\title{
Numerical Simulation of 2D Elastic Problems Using RBF-FD Method Wei $\mathrm{LI}^{1, \mathrm{a}}$, Chun-Guang $\mathrm{LI}^{2, \mathrm{~b}}$, Hong $\mathrm{ZHENG}^{1, \mathrm{c}}, \mathrm{H}$ ong-Wei GUO ${ }^{1, \mathrm{~d}}$, Zhi-Fen WANG ${ }^{2, e}$
}

\author{
${ }^{1}$ Key Laboratory of Geological Hazards on Three Gorges Reservoir Area, Ministry of Education,China \\ Three Gorges University, Yi Chang 443002, China \\ ${ }^{2}$ State Key Laboratory of Geomechanics and Geotechnical Engineering, Institute of Rock and Soil \\ Mechanics, Chinese Academy of Sciences, Wuhan, Hubei 430071, China \\ aliw9012@163.com, ${ }^{b} \mathrm{cgli@whrsm.ac.cn,}{ }^{\mathrm{C}} \mathrm{hzheng@whrsm.ac.cn,}{ }^{\mathrm{d}} \mathrm{ghway0703@163.com,}$ \\ 'zhifenwang@163.com
}

\begin{abstract}
Keywords: Meshless Method, Radial Basis Function, Finite Difference, Hermite Interpolation, Elasticity.
\end{abstract}

\begin{abstract}
This work applies the radial basis function-finite difference method (RBF-FD) for the solution of 2D elastic problems. Compared with traditional finite difference methods based on polynomial interpolation, the RBF-FD does not require a regular arrangement of nodes but can achieve high accuracy. With $\delta$-property, the boundary conditions can be easily imposed. To deal with the stress boundary conditions, the Hermite interpolation is used in calculation of the approximation function and discrete equation. The validity is examined by typical examples in the treatment of $2 \mathrm{D}$ problems in elasticity.
\end{abstract}

\section{Introduction}

Meshless method [1] is a numerical method developed in recent years. Compared with FEM, the meshless methods, which don't discrete problem domain using grid, own many advantages, for example, simple in pre-processing and adaptive analysis, easy to construct higher-order smooth shape functions, prone to expand low-dimensional problems into high-dimensional problems and solves difficult problems that the traditional numerical methods can't deal with [2], such as extremely large deformation, dynamic crack propagation, high-speed impact and so on. Therefore, the meshless method is considered a potential numerical analysis method.

Recently, dozens of meshless methods have been developed. According to the formulations, the different procedures can be divided into three categories: (1) strong-form-based collocation meshless methods, such as the finite point method [3] and hp-clouds[4]; (2) weak-form-based meshless methods, such as EFG method [5], the diffuse element method [6] and MLPG method [7]; (3) Combined weak and strong forms meshless method- MWS [7]. In these methods, the EFGM is widely used at present, which has high accuracy and good stability. Because of requiring background grid for quadrature and matrix inversions, it involves a large amount of calculation. In contrast, collocation meshless methods are truly meshless methods, and are very efficient. However, this method exist stability and convergence speed issues, and further improvement is needed.

Wright and Fornberg [9] proposed a radial basis function-finite difference method (RBF-FD), which is mainly adopted in fluid mechanics. This paper attempts to apply this method to 2D elastic problems. Compared with traditional finite difference methods based on polynomial interpolation, the RBF-FD does not require a regular arrangement of nodes but can achieve high accuracy. Due to the $\delta$-property, it is easy to impose boundary conditions. Meanwhile, in order to reduce the error arising from stress boundary conditions, the Hermite interpolation is adopted. Finally, the accuracy of the method is discussed by some classic examples. 


\section{D Elastic Problem}

\section{Equations}

Assume that $\Omega$ represents the problem domain, $\Gamma_{t}$ represents the traction boundary and $\Gamma_{u}$ represents the displacement boundary.

In plane stress problems, in terms of the displacement $u_{1}$ and $u_{2}$ the problem can be formulated in the matrix form

$$
\begin{aligned}
& \frac{E}{1-v^{2}}\left[\begin{array}{ll}
\frac{\partial^{2}}{\partial x_{1}^{2}}+\frac{1-v}{2} \frac{\partial^{2}}{\partial x_{2}^{2}} & \frac{1+v}{2} \frac{\partial^{2}}{\partial x_{1} \partial x_{2}} \\
\frac{1+v}{2} \frac{\partial^{2}}{\partial x_{1} \partial x_{2}} & \frac{\partial^{2}}{\partial x_{2}^{2}}+\frac{1-v}{2} \frac{\partial^{2}}{\partial x_{1}^{2}}
\end{array}\right]\left[\begin{array}{l}
u_{1} \\
u_{2}
\end{array}\right]=-\left[\begin{array}{l}
\overline{f_{1}} \\
f_{2}
\end{array}\right] \text { in } \Omega, \\
& \frac{E}{1-v^{2}}\left[\begin{array}{ll}
n_{1} \frac{\partial}{\partial x_{1}}+n_{2} \frac{1-v}{2} \frac{\partial}{\partial x_{2}} & n_{1} v \frac{\partial}{\partial x_{2}}+n_{2} \frac{1-v}{2} \frac{\partial}{\partial x_{1}} \\
n_{2} v \frac{\partial}{\partial x_{1}}+n_{1} \frac{1-v}{2} \frac{\partial}{\partial x_{2}} & n_{2} \frac{\partial}{\partial x_{2}}+n_{1} \frac{1-v}{2} \frac{\partial}{\partial x_{1}}
\end{array}\right]\left[\begin{array}{l}
u_{1} \\
u_{2}
\end{array}\right]=-\left[\frac{\overline{t_{1}}}{t_{2}}\right] \text { in } \Gamma_{t}, \\
& {\left[\begin{array}{l}
u_{1} \\
u_{2}
\end{array}\right]=\left[\begin{array}{l}
\overline{u_{1}} \\
u_{2}
\end{array}\right] \text { in } \Gamma_{u},}
\end{aligned}
$$

where $E$ is Young's modulusy and $v$ Poisson's ratio.

For Plane strain problems, $E=\frac{E}{1-v^{2}}, v=\frac{v}{1-v} \cdot n_{1}$ and $n_{2}$ are the direction cosines of the outward normal direction of boundary $\Gamma_{t}$.

\section{Discrete Equations}

Assuming that there are $N$ nodes in domain, $N_{t}$ nodes on $\Gamma_{t}, N_{u}$ nodes on $\Gamma_{u}, n$ nodes in $\Omega$, and $N_{\Omega}=N+N_{t}+N_{u}$.

From $(1) \sim(3)$, let $L_{i}$ be the following differential operators, $L_{0}=1, L_{1}=\frac{\partial^{2}}{\partial x_{1}^{2}}+\frac{1-v}{2} \frac{\partial^{2}}{\partial x_{2}^{2}}$, $L_{2}=\frac{1+v}{2} \frac{\partial^{2}}{\partial x_{1} \partial x_{2}}, L_{3}=\frac{\partial^{2}}{\partial x_{2}^{2}}+\frac{1-v}{2} \frac{\partial^{2}}{\partial x_{1}^{2}}, L_{4}=\frac{\partial}{\partial x_{1}}, L_{5}=\frac{\partial}{\partial x_{2}}$. Substituting those operators into (25) of the paper [9], we can obtain $c_{i j}^{k}$ corresponding to $L_{k} u\left(x_{i}\right)$ in $x_{i}$.where $k=0,1, \cdots, 5, i=1, \cdots, N$, $j=1, \cdots, n, c_{i j}^{k}=\left\{\begin{array}{l}c_{i j}^{k}, x_{j} \in\left\{x_{1}, \cdots, x_{n}\right\} \\ 0, x_{j} \notin\left\{x_{1}, \cdots, x_{n}\right\}\end{array}\right.$.

Substituting $c_{i j}^{1}, c_{i j}^{2}, c_{i j}^{3}$ into (1) results in

$$
D U=\bar{f}
$$

where $\boldsymbol{D}=\left[\begin{array}{llll}\boldsymbol{D}^{T}\left(x_{1}\right) & \boldsymbol{D}^{T}\left(x_{2}\right) & \cdots & \boldsymbol{D}^{T}\left(x_{N}\right)\end{array}\right]^{T}, \boldsymbol{D}\left(x_{i}\right)=\left[\begin{array}{llll}\boldsymbol{D}_{1}\left(x_{i}\right) & \boldsymbol{D}_{2}\left(x_{i}\right) & \cdots & \boldsymbol{D}_{N}\left(x_{i}\right)\end{array}\right]$,

$$
\boldsymbol{D}_{j}\left(x_{i}\right)=\frac{E}{1-v^{2}}\left[\begin{array}{ll}
c_{i j}^{1} & c_{i j}^{2} \\
c_{i j}^{2} & c_{i j}^{3}
\end{array}\right] i=1, \cdots, N, j=1, \cdots, N_{\Omega}, \boldsymbol{U}=\left[\begin{array}{lllllll}
u_{11} & u_{12} & u_{21} & u_{22} & \cdots & u_{N_{\Omega} 1} & u_{N_{\Omega} 2}
\end{array}\right]^{T},
$$


$\overline{\boldsymbol{f}}=\left[\begin{array}{lllllll}\bar{f}_{11} & \bar{f}_{12} & \bar{f}_{21} & \bar{f}_{22} & \cdots & \bar{f}_{N 1} & \bar{f}_{N 2}\end{array}\right]^{T}$.

Substituting $c_{i j}^{4}, c_{i j}^{5}$ into (2) yields

$T U=\bar{t}$

where $\boldsymbol{T}=\left[\begin{array}{lllll}\boldsymbol{T}^{T}\left(x_{1}\right) & \boldsymbol{T}^{T}\left(x_{2}\right) & \cdots & \boldsymbol{T}^{T}\left(x_{N_{t}}\right)\end{array}\right]^{T}, \boldsymbol{T}\left(x_{i}\right)=\left[\begin{array}{llll}\boldsymbol{T}_{1}\left(x_{i}\right) & \boldsymbol{T}_{2}\left(x_{i}\right) & \cdots & \boldsymbol{T}_{N}\left(x_{i}\right)\end{array}\right]$,

$\boldsymbol{T}_{j}\left(x_{i}\right)=\frac{E}{1-v^{2}}\left[\begin{array}{ll}n_{1} c_{i j}^{4}+n_{2} \frac{1-v}{2} c_{i j}^{5} & n_{1} v c_{i j}^{5}+n_{2} \frac{1-v}{2} c_{i j}^{4} \\ n_{2} v c_{i j}^{4}+n_{1} \frac{1-v}{2} c_{i j}^{5} & n_{2} c_{i j}^{5}+n_{1} \frac{1-v}{2} c_{i j}^{4}\end{array}\right] i=1, \cdots, N_{t}, j=1, \cdots, N_{\Omega}$,

$\overline{\boldsymbol{t}}=\left[\begin{array}{lllllll}\overline{t_{11}} & \overline{t_{12}} & \overline{t_{21}} & \overline{t_{22}} & \cdots & \overline{t_{N_{1}}} & \overline{t_{N_{t}}}\end{array}\right]^{T}$.

Substituting $c_{i j}^{0}$ into (3) gives rise to

$N U=\bar{u}$

where $\boldsymbol{N}=\left[\begin{array}{llll}\boldsymbol{N}^{T}\left(x_{1}\right) & \boldsymbol{N}^{T}\left(x_{2}\right) & \cdots & \boldsymbol{N}^{T}\left(x_{N_{u}}\right)\end{array}\right]^{T}, \boldsymbol{N}\left(x_{i}\right)=\left[\begin{array}{llll}\boldsymbol{N}_{1}\left(x_{i}\right) & \boldsymbol{N}_{2}\left(x_{i}\right) & \cdots & \boldsymbol{N}_{N}\left(x_{i}\right)\end{array}\right]$,

$\boldsymbol{N}_{j}\left(x_{i}\right)=\left[\begin{array}{cc}c_{i j}^{0} & 0 \\ 0 & c_{i j}^{0}\end{array}\right] \quad i=1, \cdots, N_{u}, j=1, \cdots, N_{\Omega}, \overline{\boldsymbol{u}}=\left[\begin{array}{lllllll}\overline{u_{11}} & \overline{u_{12}} & \overline{u_{21}} & \overline{u_{22}} & \cdots & \overline{u_{N_{u} 1}} & \overline{u_{N_{u}}}\end{array}\right]^{T}$.

Organizing $(4) \sim(6)$ leads to

$\boldsymbol{K} \boldsymbol{U}=\boldsymbol{P}$

where $\boldsymbol{K}=\left[\begin{array}{lll}\boldsymbol{D}^{T} & \boldsymbol{T}^{T} & \boldsymbol{N}^{T}\end{array}\right]^{T}, \boldsymbol{P}=\left[\begin{array}{lll}\overline{\boldsymbol{f}}^{T} & \overline{\boldsymbol{t}}^{T} & \overline{\boldsymbol{u}}^{T}\end{array}\right]^{T}$.

The stresses can be solved by

$$
\left[\begin{array}{c}
\sigma_{x}^{i} \\
\sigma_{y}^{i} \\
\tau_{x y}^{i}
\end{array}\right]=\left[\begin{array}{ccccc}
\frac{E}{1-v^{2}} c_{i 1}^{4} & \frac{E v}{1-v^{2}} c_{i 1}^{5} & \cdots & \frac{E}{1-v^{2}} c_{i N_{\Omega}}^{4} & \frac{E v}{1-v^{2}} c_{i N_{\Omega}}^{5} \\
\frac{E v}{1-v^{2}} c_{i 1}^{4} & \frac{E}{1-v^{2}} c_{i 1}^{5} & \cdots & \frac{E v}{1-v^{2}} c_{i N_{\Omega}}^{4} & \frac{E}{1-v^{2}} c_{i N_{\Omega}}^{5} \\
\frac{E}{2(1+v)} c_{i 1}^{4} & \frac{E}{2(1+v)} c_{i 1}^{5} & \cdots & \frac{E}{2(1+v)} c_{i N_{\Omega}}^{4} & \frac{E}{2(1+v)} c_{i N_{\Omega}}^{5}
\end{array}\right] \boldsymbol{U}, \quad i=1, \cdots, N_{\Omega} .
$$

\section{Improved Stress Boundary Conditions}

For the problem of the second boundary condition, the accuracy of the results obtained using the above method is not high, especially in the vicinity of the boundary. This paper adopts the Hermite interpolation to improve the accuracy. The specific process of the method is given below.

The interpolant takes the form

$$
s(x)=\sum_{j=1}^{N_{\Omega}} \lambda_{j} \phi_{j}(x)+\sum_{k=1}^{N_{t}} \gamma_{k} \frac{\partial \phi_{k}(x)}{\partial \boldsymbol{n}}+\beta
$$

where 


$$
\frac{\partial \phi_{k}(x)}{\partial \boldsymbol{n}}=n_{1} \frac{\partial \phi_{k}(x)}{\partial x_{1}}+n_{2} \frac{\partial \phi_{k}(x)}{\partial x_{2}}
$$

Referring to the paper [9], the corresponding results are acquired, and (4) (7) are adapted appropriately.

In this paper, the following examples make use of this approach to stress boundary conditions.

\section{Numerical Examples}

Two numerical examples are studied in this section. The materials used in the examples are all linear elastic with Young's modulus $E=1$ and Poisson's ratio $v=1 / 3$. The units used in this paper can be any consistent unit based on international standard unit system.

\section{Internal Pressurized Hollow Cylinder}

A hollow cylinder subjected to internal pressure (shown in Fig.1) is analyzed. The cylinder is of internal radius $a=1$, outer radius $b=5$, and internal pressure $p=1$ units. Plain strain condition is considered and the analytical solutions can be referred to [10]

$$
\begin{aligned}
& u_{r}(r)=\frac{a^{2} p r}{E\left(b^{2}-a^{2}\right)}\left[1-v+\frac{b^{2}}{r^{2}}(1+v)\right] \\
& u_{\theta}=0 \\
& \sigma_{r}(r)=\frac{a^{2} p}{b^{2}-a^{2}}\left(1-\frac{b^{2}}{r^{2}}\right) \\
& \sigma_{\theta}(r)=\frac{a^{2} p}{b^{2}-a^{2}}\left(1+\frac{b^{2}}{r^{2}}\right) \\
& \sigma_{r \theta}=0
\end{aligned}
$$

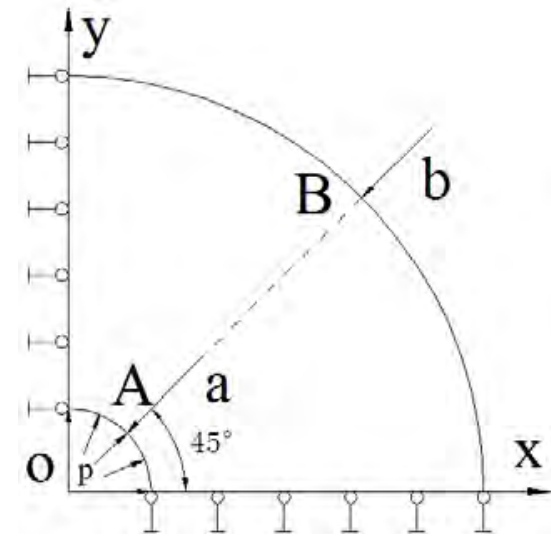

Fig. 1 A Quarter Model of Hollow Cylinder

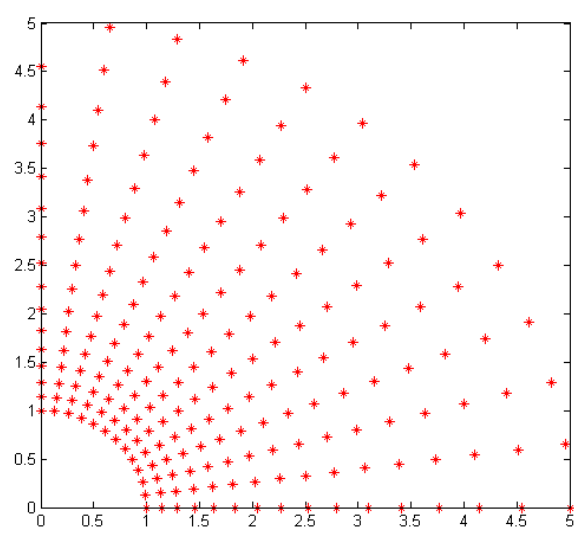

Fig. 2 Nodal Arrangement

The problem is discretized by 123 irregularly distributed nodes (shown in Fig. 2). Meanwhile the ABAQUS (adopting CPE3) and the exact solution are used to calculate the displacements and stresses at the same nodal distribution. Symmetry conditions are imposed on the left and bottom edges, the inner boundary of the hole is internal pressure $\mathrm{p}$, and the external boundary of the hole is traction free.

The result and relative error of the measured points $(A, B)$ are calculated in Table 1. The figures (Fig.3 and Fig.4) show that the numerical solutions obtained using the present method is in a very good agreement with the exact solutions and the accuracy is higher than ABAQUS. 


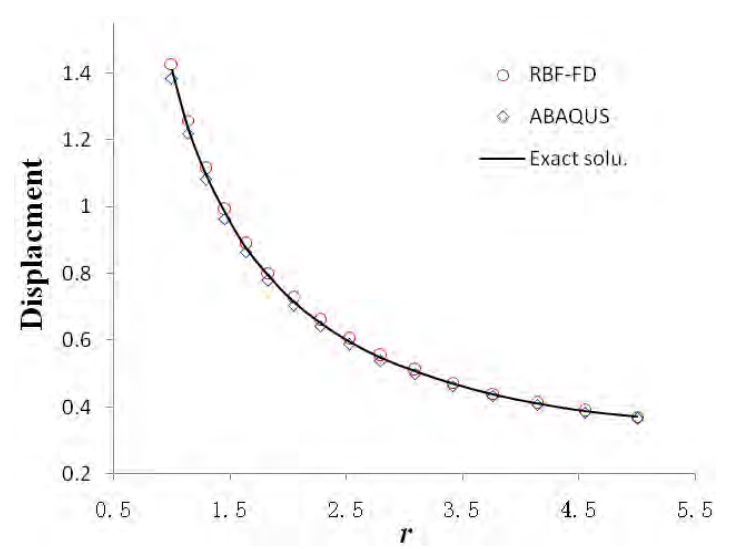

Fig.3 Displacement Distribution along Radial Line of Hollow Cylinder $\left(\theta=45^{\circ}\right)$

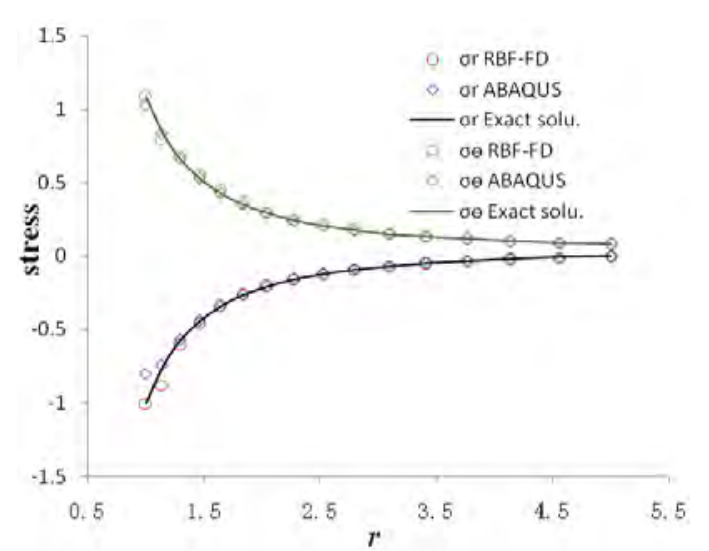

Fig.4 Stress Distribution along Radial Line of Hollow Cylinder $\left(\theta=45^{\circ}\right)$

Tab. 1 The Result and Relative Error of the Measured Points $(A, B)$

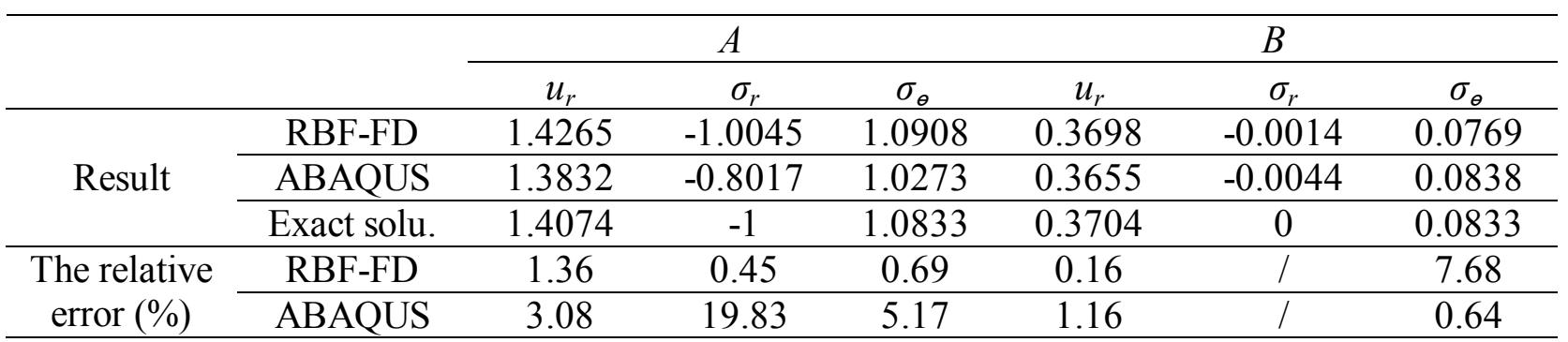

\section{Cook Skew Beam}

The Cook skew beam is also analyzed. The model and size are shown in Fig. 5. The right boundary of the beam is applied by the distribution of shear force $F=1 / 16$. The left boundary of the beam is displacement boundary condition with $u=0, v=0$.

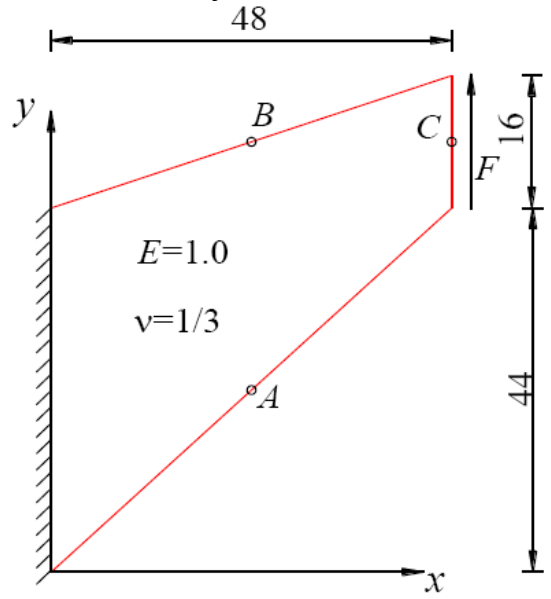

Fig. 5 Cook Skew Beam Suffered from

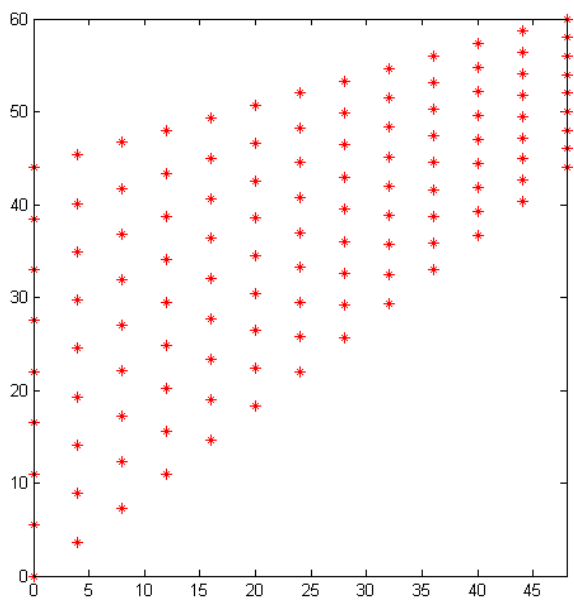

Fig. 6 Nodal Arrangement

Distributed shear force on the right

Plane stress condition is considered and traction boundary conditions are imposed on the upper and right edges with free. The domain is discretized using 117 irregularly distributed nodes, as shown in Fig. 6. Meanwhile the ABAQUS (adopting CPS3) and the reference solution are analyzed with the same nodal distribution.

In the Cook skew beam, the stress and displacement of 3 measured points $(A, B, C)$ are calculated (shown in Table 2).Compared with reference solution, it is clear that the solution of the RBF-FD method is more accuracy than the ABAQUS. 
Tab. 2 Comparison of the Stress and Displacement for 3 Measured Points

\begin{tabular}{cccc}
\hline Method & $\sigma_{\operatorname{maxA}}$ & $\sigma_{\min B}$ & $V_{C}$ \\
\hline RBF-FD & 0.2334 & -0.1969 & 23.85 \\
\hline ABAQUS & 0.2105 & -0.1712 & 22.91 \\
\hline Reference solution[11] & 0.2362 & -0.2023 & 23.96 \\
\hline
\end{tabular}

\section{Conclusion}

Radial basis function-finite difference method (RBF-FD), which is widely used in computational fluid mechanics, is introduced to solving 2D problems in elasticity. The Hermite interpolation is adopted to deal with the stress boundary conditions. Finally, the accuracy of the method is discussed by two classic examples. The following main conclusions can be obtained.

1. Using the Hermite interpolation can greatly improve the solution accuracy.

2. This method belongs to strong-form-based collocation meshless methods, which needs not domain integration, so its programming is simple and it has a higher efficiency.

3. This method has higher accuracy than the conventional finite element.

\section{Acknowledgement}

This study is supported by the National Natural Science Foundation of China, under the grant number: 11172313.

\section{References}

[1]Atluri S N, Shen S. The meshless method [J]. Forsyth: Tech. Sci. Press USA, 2002.

[2]Liu G R. Meshfree methods: moving beyond the finite element method [M]. CRC, 2009.

[3] Onate E, Idelsohn S, Zienkiewicz O C, et al. A stabilized finite point method for analysis of fluid mechanics problems [J]. Computer Methods in Applied Mechanics and Engineering, 1996, 139(1): 315-346.

[4]Duarte C A, Oden J T. Hp clouds-an hp meshless method[J]. Numerical methods for partial differential equations, 1996, 12(6): 673-706.

[5]Krysl P, Belytschko T. The Element Free Galerkin method for dynamic propagation of arbitrary 3 - D cracks [J]. International Journal for Numerical Methods in Engineering, 1999, 44(6): 767-800.

[6]Nayroles B, Touzot G, Villon P. Generalizing the finite element method: diffuse approximation and diffuse elements [J]. Computational mechanics, 1992, 10(5): 307-318.

[7]Atluri S N, Zhu T. A new meshless local Petrov-Galerkin (MLPG) approach in computational mechanics [J]. Computational mechanics, 1998, 22(2): 117-127.

[8]Gu Y T, Liu G R. A meshfree weak-strong (MWS) form method for time dependent problems[J]. Computational Mechanics, 2005, 35(2): 134-145.

[9] Wright G B, Fornberg B. Scattered node compact finite difference-type formulas generated from radial basis functions[J]. Journal of Computational Physics, 2006, 212(1): 99-123.

[10]Timoshenko S P, Goodier J N. Theory of Elasticity [M]. 3rd ed New York: McGraw-Hill,1970.

[11]Cook R D, Malkus D S, Plesha M E. Concepts and applications of finite element analysis. 3rd ed. New York: John Wiley \& Sons Inc., 1989. 